\title{
LACTANCIA MATERNA EXCLUSIVA DURANTE LOS PRIMEROS 6 MESES DE VIDA COMO FACTOR PROTECTOR PARA ASMA BRONQUIAL EN POBLACIÓN PEDIÁTRICA
}

\author{
Marisa Sotelo ${ }^{1, a}$, Juan Carlos Roque $2, a, b$, José Arturo Vargas ${ }^{2, a, d}$, \\ Joseph Alburqueque-Melgarejo ${ }^{1, c}$, Claudia Saldaña ${ }^{3, a, b}$
}

\begin{abstract}
RESUMEN
Objetivo: determinar la relación entre lactancia materna exclusiva durante los primeros 6 meses de vida y asma bronquial en población pediátrica en el hospital Nacional de la Policía en el año 2016. Materiales y métodos: se realizó un estudio observacional analítico tipo casos y controles no emparejado de razón 1 caso para 3 controles, con un total de 53 casos y 159 controles, se realizó un muestreo probabilístico aleatorio simple, con una recolección retrospectiva para las variables lactancia materna exclusiva, Prematuridad, sexo y edad. Se realizó un modelo de regresión logística bivariado y múltiple para la obtención del Odds Ratio para la fuerza de asociación. Resultados: siendo $24.53 \%$ y $44.65 \%$ de lactancia materna exclusiva para los casos y controles, se encontró una diferencia estadísticamente significativa $(p=0.009)$. Por análisis de regresión logística múltiple lactancia materna exclusiva (OR 0.41, $p=0.014$, IC $0.20-0.84$ ) presento relación para asma bronquial. Conclusiones: se encontró una relación entre lactancia materna exclusiva y Asma bronquial la cual puede ser extrapolada a la población pediátrica de la ciudad de Lima, este es el primer artículo publicado al respecto a nivel nacional, se recomiendan estudios prospectivos para determinar causalidad.
\end{abstract}

Palabras clave: Lactancia materna exclusiva, asma bronquial, leche maternizada (Fuente: DeCS BIREME).

\section{EXCLUSIVE BREASTFEEDING DURING THE FIRST 6 MONTHS OF LIFE AS A PROTECTIVE FACTOR FOR BRONCHIAL ASTHMA IN THE PEDIATRIC POPULATION}

\begin{abstract}
Objective: to determine the relationship between exclusive breastfeeding during the first 6 months of life and bronchial asthma in the pediatric population at the National Police Hospital in 2016. Materials and methods: an analytical observational study of cases and controls was not matched. ratio 1 case for 3 controls, with a total of 53 cases and 159 controls, a simple random probability sampling was performed, with a retrospective collection for the variables exclusive breastfeeding, Prematurity, sex and age. A bivariate and multiple logistic regression model was performed to obtain the Odds Ratio for the strength of association. Results: being $24.53 \%$ and $44.65 \%$ exclusive breastfeeding for cases and controls, a statistically significant difference was found $(p=0.009)$. By multiple logistic regression analysis exclusive breastfeeding (OR $0.41, p=$ 0.014 , IC 0.20-0.84) showed a relationship for bronchial asthma. Conclusions: a relationship was found between exclusive breastfeeding and bronchial asthma which can be extrapolated to the pediatric population of the city of Lima, this is the first article published in this regard at the national level, prospective studies are recommended to determine causality.
\end{abstract}

Key words: exclusive breastfeeding, bronchial asthma, formula (Source: MeSH NLM).

\section{INTRODUCCIÓN}

El asma es la enfermedad crónica de la infancia más común, con una prevalencia de $14 \%$ que va en aumento. En población pediátrica, es la principal causa, de visitas a la emergencia, hospitalizaciones y días perdidos de escuela ${ }^{1}$. Múltiples autores remontan su etiología al desarrollo de la respuesta inmunitaria desde el nacimiento a los primeros dos años de vida. Se ha observado que una microbiota intestinal balanceada y diversa predispone al desarrollo adecuado de la inmunidad, en relación a esto, se ha encontrado que los desbalances de la microbiota intestinal en las primeras semanas de vida se han asociado al desarrollo de eczema y asma en la infancia ${ }^{2} a t$ $5,13,21$, and 31 weeks postpartum (infants. La lactancia materna en los primeros 4 a 6 meses de vida ayudaría al desarrollo de una microbiota intestinal saludable al proveer bifidobacterias y bacterias acido lácticas productoras de

\footnotetext{
Universidad Ricardo palma

Universidad Científica del Sur, Departamento de Morfofisiologia

Instituto Nacional Materno Perinatal

Médico cirujano

Maestro en medicina

Estudiante de medicina

Maestro en educación

Citar como: Sotelo M, Roque JC, Vargas JA, Alburqueque-Melagarejo J, Saldaña C. Lactancia materna exclusiva durante los primeros 6 meses de vida como factor protector para asma bronquial en población pediátrica. Rev Peru Investig Matern Perinat 2020; 9(2): 21-25 DOI https://doi.org/10.33421/inmp.2020201
}

Recibido: 22-02-20 Aprobado: 30-06-20 
oligosacáridos promoviendo una composición microbial saludable ${ }^{3}$, así mismo, provee inmunoglobulinas, ácidos grasos insaturados, quimioquinas, alérgenos citoquinas inmunoactivas estimulantes de la maduración de la inmunidad mucosa del infante. ${ }^{4} \mathrm{Si}$ bien las bases teóricas y múltiples artículos han dado indicio de la relación protectora entre lactancia y asma, algunos autores muestran su discrepancia debido a la heterogeneidad de la evidencia. Se ha observado que la lactancia disminuye el riesgo de infecciones virales de tracto respiratorio bajo y se propone que esta sería esta una de los mecanismos en la que la lactancia protegería del asma, ya que las infecciones virales a ese nivel en la primera infancia predisponen al desarrollo de esta enfermedad 5 .

La situación del Perú en relación a la lactancia materna según el informe ENDES 2018 es bastante favorable en relación a la región. Se estimó que el $98,9 \%$ de los niños han lactado alguna vez dentro de los primeros 2 años de vida, en comparación al $21-39 \%$ de lactancia observada en Sudamérica y el Caribe según Unicef ${ }^{6}$. Así mismo el $66.4 \%$ de los niños menores de 6 meses recibieron lactancia materna. Sin embargo un $51,6 \%$ de los niños recibieron leche maternizada ante de empezar a lactar en el área urbana ${ }^{7}$.

La presente investigación pretende determinar la relación entre la lactancia materna durante los primeros 6 meses y el asma bronquial en una población pediátrica de Lima metropolitana.

\section{MATERIALES Y MÉTODOS}

Diseño: Se realizó un diseño de estudio tipo observacional, analítico, retrospectivo, casos y controles no emparejado de una proporción de 3 controles para 1 caso. La recolección de los datos se llevó a cabo en el hospital nacional de la policía nacional del Perú durante el año 2017, se realizó una recolección de datos de pacientes atendidos entre los años 2016.

La población objetivo fueron aquellos pacientes pediátricos con edades entre 3 a 12 años diagnosticados de asma atendidas en consulta externa del servicio de pediatría general que cumplieron con los criterios de selección, donde los casos fueron aquellos pacientes con asma bronquial y los controles aquellos pacientes sin asma bronquial. Como factor de exposición se tomó a la lactancia materna exclusiva.

Procedimientos y variables: Dentro de los criterios de selección para los casos y controles se tomaron como criterios de inclusión historia clínica con datos completos para las variables estudiadas; como criterios se exclusión se tomó a la parálisis cerebral infantil, malformaciones de la vía respiratoria, digestiva superior, rinitis alérgica, alergia alimentaria y presencia de pólipos nasales.

La variable independiente en el estudio fue lactancia materna exclusiva, cuyo instrumento diagnóstico fue la entrevista durante la anamnesis registrada en la historia clínica la cual fue tomada por especialistas del servicio de consulta externa de pediatría. La variable dependiente, asma bronquial empleo como instrumento diagnostico al espirómetro de acuerdo a las recomendaciones de la guía GINA. El cual fue dado por especialistas de consulta externa de pediatría. Como otras variables se registró la edad en años del paciente al momento del diagnóstico del asma bronquial, género, grado de instrucción de la madre y lactancia materna exclusiva, las cuales fueron tomadas de la anamnesis y antecedentes presentes en la fuente de información secundaria, la historia clínica.

Los datos de cada una de las variables mencionadas fueron registrados en una ficha de recolección de datos para cada sujeto de estudio las cuales se almacenaron para su posterior vaciado y análisis estadístico.

Población y Muestra: Para el cálculo del tamaño muestral se tomó un intervalo de confianza del 95\%, una potencia estadística del $80 \%$, una proporción de $1: 3$ para casos y controles respectivamente, tomaron los resultados obtenidos por Ricra et $\mathrm{al}^{8}$ con una proporción de casos expuestos de $44.44 \%$, proporción de controles expuestos $66.67 \%$, se empleó el paquete epidemiológico OpenEpi de acceso libre para el cálculo muestral donde se obtuvo un total de 212 pacientes, siendo 53 casos y 159 controles respectivamente empleando la fórmula de Kelsey. Se realizó un muestreo de tipo probabilístico unietapico donde se estratifico a la población en pacientes con Asma bronquial y sin asma bronquial, se realizó un muestreo aleatorio simple en cada uno de los estratos, sin reposición.

Cuestiones Éticas: Se respetó la confidencialidad de los datos personales de cada sujeto de estudio, se contó con la aprobación de la oficina de capacitación y docencia del Hospital, así como la aprobación por parte del consejo de facultad de la universidad Ricardo Palma.

Análisis estadístico: Las fichas de recolección de datos fueron vaciadas a una hoja de cálculo Excel, donde por medio del programa Stat-Tranfer, fueron transportados al paquete estadístico STATA versión 15 para su posterior análisis estadístico.

Los estadísticos descriptivos se presentaran para los casos y los controles reportándose la media y desviación estándar para la variable cuantitativa edad, así como la frecuencia absoluta y relativa para cada variable cualitativa, esto es sexo, prematuridad y lactancia materna exclusiva.

Para la estadística inferencial se realizó la prueba estadística no paramétrica chi cuadrado para contrastar las variables cualitativas y una prueba de contraste de medias para las variables cuantitativas respecto a los casos y controles, posteriormente se realizará un modelo de regresión logística bivariado, para la obtención del Odds ratio e intervalos de confianza al 95\%. 
Para concluir se ejecutará una modelo de regresión logística multivariado para la obtención de un Odds ratio ajustado con sus respectivos intervalos de confianza al $95 \%$, con aquellas variables que son consideradas como factores de riesgo para el Asma bronquial.

\section{RESULTADOS}

Del total muestral de 212 pacientes pediátricos ninguno de los pacientes estudiados presento datos perdidos para las variables estudiadas, 53 fueron asmáticos y 159 no asmáticos, la media y desviación estándar de edad para ambos grupos siendo 9.56 +/- 2.18 y 9.3 $+/-2.17$ respectivamente, sin presentar diferencias estadísticamente significativas. El porcentaje de sexo masculino fue superior en los pacientes no asmáticos $55.97 \%$, en relación a los pacientes asmáticos 50,94\%, sin encontrarse diferencia estadísticamente significativa entre los grupos con un $p=0.524$. El porcentaje de lactancia materna exclusiva fue superior en pacientes no asmáticos $44.65 \%$, en relación a los pacientes asmáticos $24.53 \%$, encontrándose una diferencia estadísticamente significativa entre los grupos con un $p=0.009$. El porcentaje de prematuridad fue superior en pacientes asmáticos $11.32 \%$, en relación a los pacientes no asmáticos $7.55 \%$, sin encontrarse diferencia estadísticamente significativa entre los grupos con un $p=0.393$ (tabla 1 ).

Tabla 1. Análisis univariado

\begin{tabular}{lccc}
\hline & $\begin{array}{c}\text { Controles } \\
(\mathrm{N}:) \%(\mathrm{n})\end{array}$ & $\begin{array}{c}\text { Casos } \\
(\mathrm{N}:) \%(\mathrm{n})\end{array}$ & Valor $\mathrm{p}$ \\
\hline $\begin{array}{l}\text { Lactancia materna } \\
\text { exclusiva }\end{array}$ & $44.65 \%(71)$ & $24.53(13)$ & 0.009 \\
Prematuridad & $7.55 \%(12)$ & $11.32 \%(6)$ & 0.393 \\
Sexo masculino & $55.97 \%(89)$ & $50.94 \%(27)$ & 0.524 \\
\hline
\end{tabular}

Valores p obtenidos de la prueba no paramétrica Chi cuadrado.

Se realizó un modelo de regresión logística bivariado para las variables sexo (OR 0.82, $p=0.524$, IC 0.44-1.53) lactancia materna exclusiva (OR $0.4, P=0.011$, IC 0.20 0.81 ), prematuridad (OR 1.56, $p=0.396, I C \quad 0.56-4.40$ ), solo la variable lactancia materna exclusiva fue la única variable que presento significancia estadística (tabla 2).

Tabla 2. Análisis con regresión logística bivariado

\begin{tabular}{lccc}
\hline Variables & $\begin{array}{c}\text { Odds } \\
\text { Ratio }\end{array}$ & P valor & $\begin{array}{c}\text { Intervalo de } \\
\text { confianza }\end{array}$ \\
\hline Lactancia materna exclusiva & 0.40 & 0.011 & $0.20-0.81$ \\
\hline Prematuridad & 1.56 & 0.396 & $0.56-4.40$ \\
Sexo Masculino & 0.82 & 0.524 & $0.44-1.53$ \\
\hline
\end{tabular}

Se realizó un modelo de regresión logística múltiple para ajustar el Odds ratio de ERGE por variables que presentan un efecto demostrado sobre el asma bronquial, sexo (OR 0.78, $p=0.447, I C$ 0.41-1.48) lactancia materna exclusiva (OR 0.41, P=0.014, IC 0.20-0.84), prematuridad
(OR 1.31, $p=0.624$, IC 0.45-3.81) (tabla 3) las variable lactancia materna fue la única que presento significancia estadística.

Tabla 3. Análisis con regresión logística Múltiple

\begin{tabular}{lccc}
\hline Variables & $\begin{array}{c}\text { Odds } \\
\text { Ratio }\end{array}$ & P valor & $\begin{array}{c}\text { Intervalo de } \\
\text { confianza }\end{array}$ \\
\hline Lactancia materna exclusiva & 0.41 & 0.014 & $0.20-0.84$ \\
Prematuridad & 1.31 & 0.624 & $0.45-3.81$ \\
Sexo Masculino & 0.78 & 0.447 & $0.41-1.48$ \\
\hline
\end{tabular}

\section{DISCUSIÓN}

El presente estudio caso-control demostró una relación entre la lactancia materna exclusiva durante los primeros 6 meses de vida y el desarrollo de asma en población pediátrica. Se encontró una prevalencia elevada de asma en pacientes que no recibieron lactancia materna en comparación con las que si la recibieron. Este hallazgo confirma los resultados de varios estudios este tipo, como los realizados por Huo, $X$ et al, Mai, $X$ et al, Lee, $S$ et al, Takemura $\mathrm{Y}$. et al, Song, $\mathrm{N}$ et al, donde reportaron que la lactancia materna representaba un factor protector para el desarrollo de asma en población pediátrica ${ }^{9-13}$. Este es el primer estudio a nivel nacional que evalúa esta asociación.

El presente estudio cuenta con algunas limitaciones propias del diseño. Una de ellas es que se trata de un estudio unicéntrico. Asimismo, la información utilizada fue generada a partir de fuentes secundarias. Nuestro estudio no evaluó la relación entre asma en edad pediátrica con otras variables como el uso de antibióticos por la madre, la dieta materna, la presencia de alergias en la madre, o la presencia de gestaciones múltiples, siendo estas variables de vital importancia, puesto que estas pueden influir en el desarrollo de asma en niños e interferir con los resultados ${ }^{14-17}$. No se evalúo el efecto de la lactancia materna exclusiva en el desarrollo de otras patologías como rinitis alérgica, dermatitis atópica, alergia a alimentos, teniendo en cuenta que varios estudios como los de Friedman $\mathrm{N}$ et al, Munblit $\mathrm{D}$ et al, Kull I et al postulan a la lactancia materna exclusiva como un factor protector para esas patologías ${ }^{14,18,19}$. Tampoco se evaluó la presencia de infecciones virales en lactantes como causa de asma puesto que estas suponen un factor de riesgo importante para el desarrollo de asma en edades posteriores ${ }^{20}$.

Nuestro estudio muestra evidencia de la asociación entre la lactancia materna exclusiva y el desarrollo de asma en niños, y se respalda con múltiples estudios de alta confiabilidad realizados previamente, como las revisiones sistemáticas y meta-análisis de Lodge $C$ et al y Dogaru $C$ et $a^{21,22}$.

Muchos estudios mencionan la ventaja que tiene la lactancia materna exclusiva durante los seis primeros meses con respecto al uso de fórmulas maternizadas o de leche de vaca sobre el desarrollo de asma. También 
señalan que la leche materna tiene una gran cantidad de proteínas, entre estas citoquinas, quimiocinas, alergenos e inmunoglobulinas, muchas de las cuales funcionan como immunomoduladores como en el caso del TGFbeta, cuya presencia ha sido implicada en el desarrollo y maduración del sistema inmunológico del lactante. Del mismo modo, la lactancia exclusiva confiere protección contra infecciones respiratorias virales durante la infancia.

También se menciona que la lactancia materna permite una interacción entre el microbioma de la madre y del lactante, lo que confiere protección contra enfermedades alérgicas, como el asma ${ }^{4,14,17,23,24}$.

Sin embargo, algunos estudios como el Ek. Weronica. et al sostienen que la lactancia materna podría no tener efecto en el desarrollo de asma. Se cree que esta inconsistencia entre estudios se debe a la heterogeneidad de estos, errores propios del diseño o la presencia de otros factores no evaluadores correctamente. Asimismo, esta inconsistencia podría explicarse en el método diagnóstico que se utilizó para asma, pudiendo sobreestimarse su diagnóstico en niños con infecciones respiratorias virales ${ }^{25}$. Se requieren estudios posteriores para esclarecer la evidencia de esta asociación. El presente estudio puede generalizarse en poblaciones pediátricas.

En conclusión, el presente artículo demuestra una relación entre la lactancia materna exclusiva durante los primeros seis meses y el desarrollo de asma en población pediátrica. Los resultados pueden generalizarse a poblaciones pediátricas de Lima metropolitana.

Financiamiento: Autofinanciado.

Conflicto de interés: Los autores declaran no tener algún conflicto de intereses

\section{REFERENCIA BIBLIOGRÁFICAS}

1. Naja AS, Permaul P, Phipatanakul W.Taming Asthma in School-Aged Children: A Comprehensive Review. The Journal of Allergy and Clinical Immunology: In Practice. 2018; 6(3): 726-735. doi:10.1016/j.jaip.2018.01.023

2. Galazzo G, van Best N, Bervoets L, Dapaah IO, Savelkoul $\mathrm{PH}$, Hornef MW, Mommers M. Development of the Microbiota and Associations With Birth Mode, Diet, and Atopic Disorders in a Longitudinal Analysis of Stool Samples, Collected From Infancy Through Early Childhood. Gastroenterology. 2020. doi:10.1053/j.gastro.2020.01.024

3. Martín $\mathrm{R}$, Olivares $\mathrm{M}$, Marín ML, Fernández L, Xaus J, Rodríguez JM. Probiotic Potential of 3 Lactobacilli Strains Isolated From Breast Milk. Journal of Human Lactation. 2005;21(1):8-17. doi:10.1177/0890334404272393

4. Oddy WH. Breastfeeding, Childhood Asthma, and Allergic Disease. Annals of Nutrition and Metabolism. 2017; 70(2):2636. doi:10.1159/000457920

5. Castro-Rodriguez JA, Forno E, Rodriguez-Martinez CE, Celedón JC. Risk and Protective Factors for Childhood
Asthma: What Is the Evidence? The Journal of Allergy and Clinical Immunology: In Practice. 2016;4(6):1111-1122. doi:10.1016/j.jaip.2016.05.003

6. Lactancia materna https://www.unicef.org/lac/media/1886/ file/PDF\%20Lactancia\%20materna.pdf

7. Capítulo 10: Lactancia y nutrición de niñas, niños y mujeres https://www.inei.gob.pe/media/MenuRecursivo/ publicaciones_digitales/Est/Lib1656/pdf/cap010.pdf

8. Ricra R, Roque JC, Alburqueque-Melgarejo J, Ricra P, Saldaña C. Gastroesophageal reflux disease as a factor associated with bronchial asthma in the pediatric population. Rev Fac Med Hum. 2020; 20(2):281-286. DOI 10.25176/RFMH.v20i2.2918

9. Huo X, Chu S, Hua L, Bao Y, Du L, Xu J, Zhang J. The effect of breastfeeding on the risk of asthma in high-risk children: a case-control study in Shanghai, China. BMC Pregnancy and Childbirth. 2018;18(1). doi:10.1186/s12884-018-1936-5

10. Bener A, Ehlayel MS, Alsowaidi S, Sabbah A. Role of breast feeding in primary prevention of asthma and allergic diseases in a traditional society. Eur Ann Allergy Clin Immunol. 2007;39(10):337-43.

11. Lee SY, Kwon JW, Seo JH, Song YH, Kim BJ, Yu J, Hong SJ. Prevalence of Atopy and Allergic Diseases in Korean Children: Associations with a Farming Environment and Rural Lifestyle. International Archives of Allergy and Immunology. 2012;158(2):168-174. doi:10.1159/000330820

12. Takemura Y. Relation between Breastfeeding and the Prevalence of Asthma: The Tokorozawa Childhood Asthma and Pollinosis Study. American Journal of Epidemiology. 2001;154(2):115-119. doi:10.1093/aje/154.2.115

13. Song N, Mohammed S, Zhang J, Wu J, Fu C, Hao S, Yan X. Prevalence, severity and risk factors of asthma, rhinitis and eczema in a large group of Chinese schoolchildren. Journal of Asthma. 2013; 51(3): 232-242

14. Friedman NJ, Zeiger RS. The role of breast-feeding in the development of allergies and asthma. Journal of Allergy and Clinical Immunology. 2005;115(6):1238-1248. doi:10.1016/j. jaci.2005.01.069

15. Al-Makoshi A, Al-Frayh A, Turner S, Devereux G. Breastfeeding Practice and Its Association with Respiratory Symptoms and Atopic Disease in 1-3-Year-Old Children in the City of Riyadh, Central Saudi Arabia. Breastfeeding Medicine. 2013:8(1):127-133. doi:10.1089/bfm.2011.0137

16. Mai XM, Becker AB, Sellers EAC, Liem JJ, Kozyrskyj AL. The relationship of breast-feeding, overweight, and asthma in preadolescents. Journal of Allergy and Clinical Immunology. 2007;120(3): 551-556. doi:10.1016/j.jaci.2007.05.004

17. Jeurink PV, Knipping $K$, Wiens $F$, et al. Importance of maternal diet in the training of the infant's immune system during gestation and lactation. Crit Rev Food Sci Nutr. 2019;59(8):1311-1319. doi: 10.1080/10408398.2017.

18. Kull I. Breast feeding and allergic diseases in infants-a prospective birth cohort study. Archives of Disease in Childhood. 2002; 87(6):478-481. doi:10.1136/adc.87.6.478

19. Munblit D, Verhasselt V. Allergy prevention by breastfeeding. Current Opinion in Allergy and Clinical Immunology. 2016;16(5):427-433. doi:10.1097/aci.0000000000000303

20. Holgate ST, Wenzel S, Postma DS, Weiss ST, Renz H, Sly PD. Asthma. Nature Reviews Disease Primers. 2015;15025. doi:10.1038/nrdp.2015.25

21. Lodge C, Tan D, Lau M, Dai X, Tham R, Lowe A, Dharmage S. Breastfeeding and asthma and allergies: a systematic 
review and meta-analysis. Acta Paediatrica. 2015;104:3853. doi:10.1111/apa.13132

22. Dogaru CM, Nyffenegger D, Pescatore AM, Spycher BD, Kuehni CE. Breastfeeding and Childhood Asthma: Systematic Review and Meta-Analysis. American Journal of Epidemiology. 2014;179(10):1153-1167. doi:10.1093/aje/ kwu072

23. Hoppu U, Kalliomaki M, Laiho K, Isolauri E. Breast milk immunomodulatory signals against allergic diseases. Allergy. 2001;56(s67): 23-26. doi:10.1034/j.1398-9995.2001.00908.x

24. Caudri D, Savenije OEM, Smit HA, Postma DS, Koppelman $\mathrm{GH}$, Wijga $\mathrm{AH}$, de Jongste JC. Perinatal risk factors for wheezing phenotypes in the first 8 years of life. Clinical \& Experimental Allergy. 2013; 43(12):1395-1405. doi:10.1111/ cea. 12173
25. Weronica EK, Torgny K, Azuaje C, Rask-Andersen M, Johansson $\AA$. Breastfeeding and risk of asthma, hay fever and eczema. Journal of Allergy and Clinical Immunology. 2017; DOI: 10.1016/j.jaci.2017.10.022

\section{Correspondencia:}

Juan Carlos Roque

Dirección: Madrid 100 Pueblo Libre

Teléfono: 945558094 